WS6-P04

\title{
Thickness Variations in Layered Subsurface Models - Effects on Simulated MASW
}

\author{
S. Bignardi* (University of Ferrara), G. Santarato (University of Ferrara) \& \\ N. Abu Zeid (University of Ferrara)
}

\section{SUMMARY}

Seismic surface wave methods allow to retrieve the shallow subsurface shear wave velocity. Among these, the "Multi-channel Analysis of Surface Waves" is to date one of the most widely adopted non-invasive active-source approaches in the professional world for the evaluation of the stiffness properties of the ground for geotechnical engineering purposes. The method utilizes the dispersive nature of surface waves by constructing the dispersion curve which is then inverted to obtain the shear wave velocity profile. Dispersion curves generation typically requires the transformation of the recorded seismograms into the frequency-velocity ( $\mathrm{f}-\mathrm{V}$ ) domain. Commercially available inversion algorithms assume the subsurface model as a stack of homogeneous parallel layers, but unfortunately this may lead to misleading results if the actual soil profile is far from the assumed 1D geometry. We investigate the effects of lateral heterogeneities due to variations of layer thicknesses on the $\mathrm{f}-\mathrm{V}$ spectrum to assess the limitations of the $1 \mathrm{D}$ approach and thus to judge the reliability of such surface waves interpretation 


\section{Introduction}

Seismic surface wave methods gained popularity during last decades to retrieve the shallow subsurface shear wave velocity (Vs). Among these, the "Multi-channel Analysis of Surface Waves" (MASW) method (Park et al., 1999), is to date one of the most widely adopted non-invasive activesource approaches in the professional world for the evaluation of the stiffness properties of the ground for geotechnical engineering purposes. The method utilizes the dispersive nature of surface waves excited by an active source and recorded by a receiver array along a profile on the ground surface. Surface waves propagation allows for the construction of the dispersion curve which is then inverted to obtain the shear wave velocity profile (Socco and Strobbia, 2004). Dispersion curves generation typically requires the transformation of the recorded seismograms into the frequency-velocity ( $\mathrm{f}-\mathrm{V}$ ) domain, where experimental dispersion curves of the propagating modes are extracted by locating the local maxima during the so-called picking operation. Commercially available inversion algorithms assume the subsurface model as a stack of homogeneous parallel layers, so that they can only capture the vertical distribution of elastic properties of the subsurface model. As a matter of fact, this may lead to misleading results if the actual soil profile is far from the assumed 1D geometry. Up to date there is a growing trend toward applications of MASW for spatially 2-D imaging and many attempts are being done to mitigate the 1-D limitation, still retaining the well-known 1D approach, (Boiero and Socco, 2010; Vignoli et al., 2011) or by a realistic subsurface modeling and taking advantage of the full waveform approach (Bignardi et al. 2012; Tran and Hiltunen, 2012) to overcome the 1D assumption. We investigate the effects of lateral heterogeneities due to variations of layer thicknesses on the $\mathrm{f}-\mathrm{V}$ spectrum to assess the limitations of the 1D approach and thus to judge the reliability of such surface waves interpretation. To do so, we investigate the vertical component of accelerations computed by a three-dimensional elastodynamic solver based on the Spectral Element Method (Komatitsch and Tromp, 1999) and compare the obtained f-V transform with theoretical dispersion modes (Herrmann 2013) for the asymptotic left and right 1D earth. We assume wave train generated by an impulsive, point source recorded by arrays with $1 \mathrm{~m}$ channel spacing and $30 \mathrm{~m}$ offset. We show that 24 receivers are not enough to allow mode separation and by consequence they are barely sensible to lateral variations. Then we investigate a 48 channels array. The case presented here as an example consists of a normally dispersive, varying thickness layer over a half space with a low acoustic impedance contrast. Effects due to a vertical/sloped interface variation together with the effects due to reciprocal location of the slope and of the geophone array are considered and are shown in figures 1 and 2 .
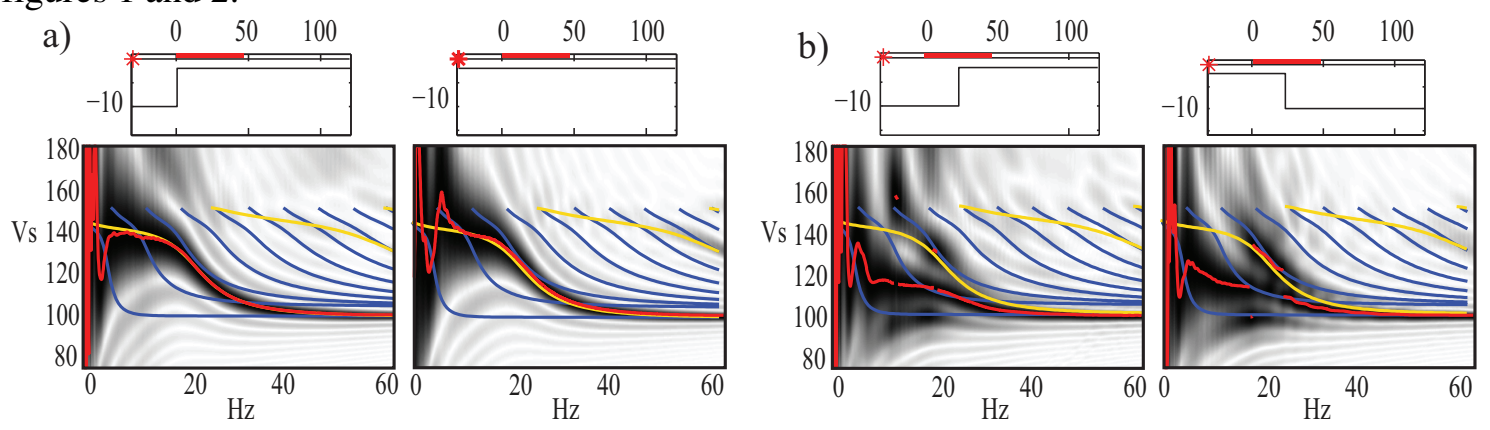

Figure 1 The $f$ - $V$ plot of different models is compared to theoretical modes for the asymptotic left flat model with interface $10 \mathrm{~m}$ depth (blue lines) and the asymptotic right flat model $2 \mathrm{~m}$ depth (yellow). Spectral maximum is highlighted in red. a) comparison of a step model against a flat model with the same local subsoil under receivers. b) example of direct and inverse shots in presence of a lateral heterogeneity. Note the different scale exaggeration in models a). 

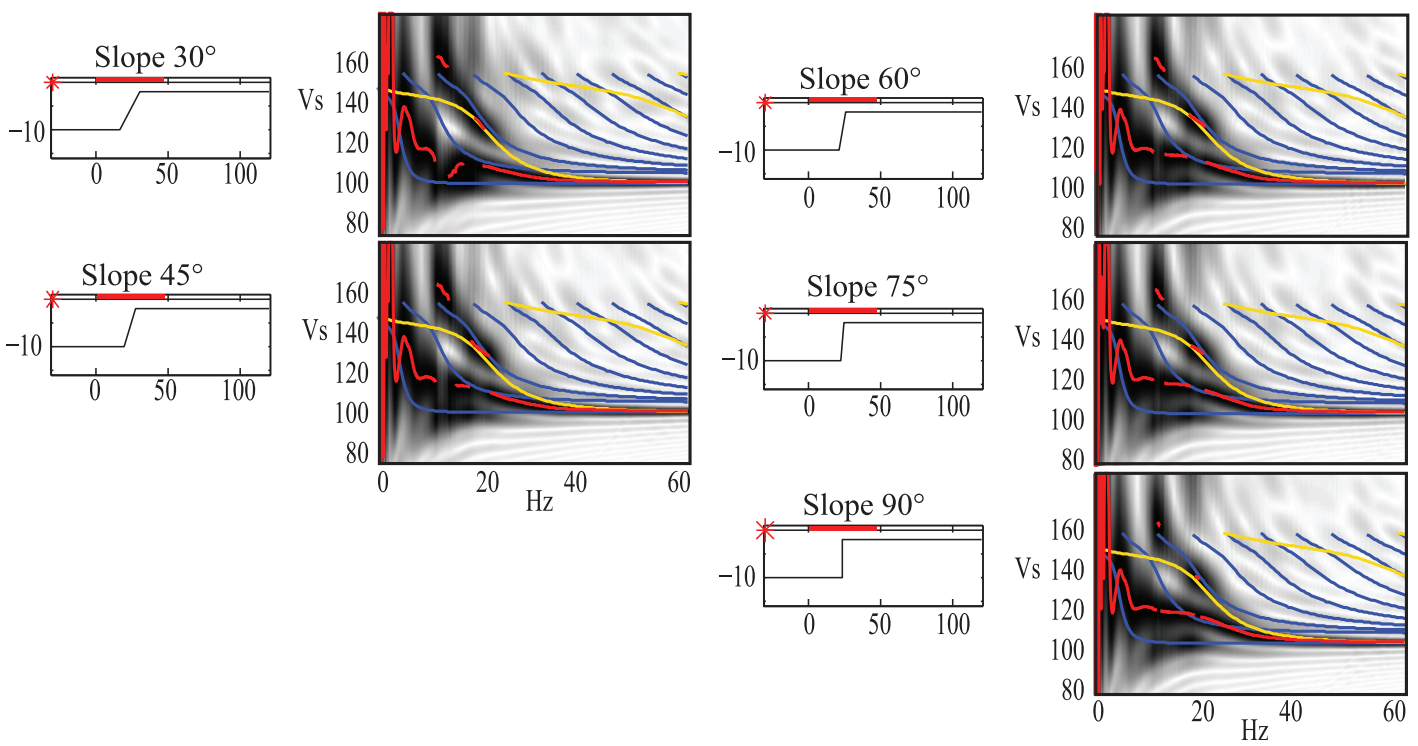

Figure 2 Following the same notation of fig. 1 , the $f$-V of different sloping modes is shown as function of the slope angle.

\section{Conclusions}

We discussed the effects of a sloping interface on the $\mathrm{f}-\mathrm{V}$ of a classical MASW setup.

The obtained $\mathrm{f}-\mathrm{V}$ compared to the multi-modal Rayleigh wave dispersion for flat layered model curves showed that when considering a $90^{\circ}$ anomaly centered on the first receiver the $f-\mathrm{V}$ is comparable to the one of a pure 1D model, i.e. the array presents a weak lateral sensitivity. When the $90^{\circ}$ anomaly is centered to the array, the $\mathrm{f}-\mathrm{V}$ pattern depends also on which side of the array the source is located. When a gentle slope is introduced below the array, $\mathrm{f}-\mathrm{V}$ is almost insensible up to an slope angle $\beta<15^{\circ}$. When angle exceeds $30^{\circ}$, the picking leads to a misleading dispersion curve but the effect is weakly depending on the slope angle, i.e. the slope is responsible only for $2^{\text {nd }}$ order effects.

\section{References}

Bignardi, S., Fedele, F., Yezzi, A.J., Rix, G. J. and Santarato G. [2012]: Geometric seismic wave inversion by the boundary element method. Bulletin of the Seismological Society of America, 102, 802-811.

Boiero, D. and Socco, L.V. [2010] Retrieving lateral variations from surface waves dispersion curves. Geophysical Prospecting, 58, 977-996.

Herrmann, R.B. [2013] Computer Programs in Seismology: An Evolving Tool for Instruction and Research. Seismological Research Letters, 84 (6).

Komatitsch, D. and Tromp, J. [1999] Introduction to the spectral element method for threedimensional seismic wave propagation. Geophysical Journal International, 139, 806-822.

Park, C.B., Miller, R.D. and Xia, J. [1999] Multichannel analysis of surface waves. Geophysics, 64 (3), 800-808.

Socco, L.V. and Strobbia, C. [2004] Surface-wave method for near-surface characterization: a tutorial. Near Surface Geophysics, 2, 165-185.

Tran K.T. and Hiltunen D.R. [2012] Shear Wave Velocity via Inversion of Full Waveforms. Proceedings of Geocongress 2012, Oakland (CA). ASCE Geotechnical Special Publication, 225, pp. 2766-2775.

Vignoli, G., Strobbia, C., Cassiani, G. and Vermeer, P. [2011] Statistical multioffset phase analysis for surface-wave processing in lateral varying media. Geophysics, 76 (2), U1--U11. 